\title{
Pediatric Chronic Critical Illness: Protocol for a Scoping Review
}

David Zorko $^{1}$, MD; James Dayre McNally ${ }^{2,3}, \mathrm{MD}$, PhD; Bram Rochwerg ${ }^{4,5,6}$, MD, MSc; Neethi Pinto ${ }^{7}$, MS, MD; Rachel Couban $^{8}$, MA, MISt; Katie O'Hearn², MSc; Karen Choong ${ }^{1,5,6}$, BCh, MB, MSc

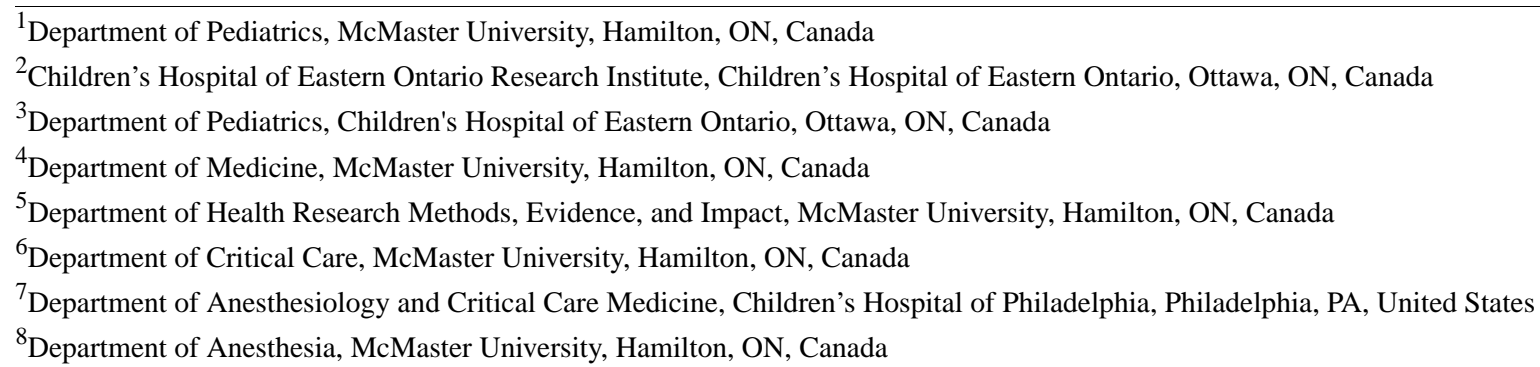

\section{Corresponding Author:}

David Zorko, MD

Department of Pediatrics

McMaster University

Room 3E20

1280 Main Street West

Hamilton, ON, L8N 3 Z5

Canada

Phone: 19055212100 ext 76651

Fax: 19055708973

Email: david.zorko@medportal.ca

\section{Abstract}

Background: Improvements in the delivery of intensive care have increased survival among even the most critically ill children, thereby leading to a growing number of children with chronic complex medical conditions in the pediatric intensive care unit (PICU). Some of these children are at a significant risk of recurrent and prolonged critical illness, with higher morbidity and mortality, making them a unique population described as having chronic critical illness (CCI). To date, pediatric CCI has been understudied and lacks an accepted consensus case definition.

Objective: This study aims to describe the protocol and methodology used to perform a scoping review that will describe how pediatric CCI has been defined in the literature, including the concept of prolonged PICU admission and the methodologies used to develop any existing definitions. It also aims to describe patient characteristics and outcomes evaluated in the included studies.

Methods: We will search four electronic databases for studies that evaluated children admitted to any PICU identified with CCI. We will also search for studies describing prolonged PICU admission, as this concept is related to pediatric CCI. Furthermore, we will develop a hybrid crowdsourcing and machine learning (ML) methodology to complete citation screening. Screening and data abstraction will be performed by 2 reviewers independently and in duplicate. Data abstraction will include the details of population definitions, demographic and clinical characteristics of children with CCI, and evaluated outcomes.

Results: The database search, crowd reviewer recruitment, and ML algorithm development began in March 2021. Citation screening and data abstraction were completed in April 2021. Final data verification is ongoing, with analysis and results anticipated to be completed by fall 2021.

Conclusions: This scoping review will describe the existing or suggested definitions of pediatric CCI and important demographic and clinical characteristics of patients to whom these definitions have been applied. This review's results will help inform the development of a consensus case definition for pediatric CCI and set a priority agenda for future research. We will use and demonstrate the validity of crowdsourcing and ML methodologies for improving the efficiency of large scoping reviews.

International Registered Report Identifier (IRRID)： DERR1-10.2196/30582

(JMIR Res Protoc 2021;10(10):e30582) doi: 10.2196/30582 


\section{KEYWORDS}

pediatrics; critical care; intensive care units; chronic critical illness; research design

\section{Introduction}

\section{Background}

Over the past two decades, the increased survival of even the most critically ill children is greatly attributed to the improvements in the delivery of intensive care [1]. An unintended consequence of this success has been a shift in the population of patients admitted to the pediatric intensive care unit (PICU), with an increasing number of children with chronic or complex medical conditions and significant long-term morbidities following critical illness [1-4]. There is a growing recognition that a subset of pediatric critical illness survivors experience persistent multiorgan system dysfunction and functional morbidities following critical illness that subsequently render them with either a prolonged need for critical care support as inpatients or dependence on medical technology to be cared for as outpatients [5-8]. These children are increasingly recognized as a uniquely high-risk PICU population, also referred to as children with chronic critical illness (CCI) $[4,6]$.

Despite being a uniquely high-risk population in the PICU, research on pediatric CCI remains limited. This patient population has been understudied, largely because of the lack of an accepted consensus case definition. The limited research to date, using variable definitions, suggests that the prevalence of children with CCI is increasing [1,2] and that these children have relatively higher morbidity and mortality rates after critical illness $[6,7,9]$. These convergent and complex issues exert significant strain on the health care system, health care providers, and caregivers [10-12]. To position the field of pediatric CCI research for systematically evaluating this important patient population, a consistent approach is needed with respect to the population that is being described and studied. Only then is it possible to determine modifiable risk factors for poor patient outcomes, and develop and evaluate interventions to improve the care and survivorship of this important PICU patient population.

\section{Objectives}

Given that we expect a heterogeneous and complex body of work, we have used a scoping review methodology to explore and describe the nature of pediatric CCI research $[13,14]$. Our primary aim is to evaluate how pediatric CCI is defined in the literature, including concepts such as prolonged or long-stay PICU admission, as it has been proposed that prolonged PICU admissions are important qualifiers for pediatric CCI $[4,6]$. The secondary aims of this scoping review are to describe the methodologies used to develop and validate any existing definitions of pediatric CCI. We will also seek to describe the prevalence of CCI in the PICU based on existing definitions and describe the key demographic and clinical characteristics of the patient populations studied. Finally, we describe the nature of the reported outcomes in children with CCI.

\section{Methods}

\section{Protocol}

This is an original scoping review following the standard methodology proposed by Arksey and O'Malley [15] and elaborated upon others $[13,16]$. This protocol is reported in accordance with the Preferred Reporting Items for Systematic Reviews and Meta-Analyses extension for scoping reviews [17]. We uploaded the protocol as a preprint to the Open Science Framework on February 1, 2021 [18]. We plan to document protocol amendments in the Open Science Framework with the date, description, and rationale. Patients and the public were not involved in the design, conduct, reporting, or dissemination plans of this research.

\section{Eligibility Criteria}

\section{Types of Participants or Population}

We will include studies that evaluated critically ill children (ie, $<18$ years old) admitted to any PICU, explicitly identified with $C C I$. We will also include studies that evaluated prolonged, protracted, chronic, or long-stay PICU admission, as this concept has been identified as an important qualifier for pediatric CCI. However, we excluded records if they (1) evaluated adult or neonatal intensive care unit populations only, or included children among these populations but did not report separate data for children; (2) evaluated pediatric patients in intermediate care, step-down, high-dependency, or chronic ventilator or respiratory units; and (3) did not include or reference a definition of pediatric CCI or prolonged PICU admission, as applicable to the study (eg, as a case definition in a prevalence study).

\section{Types of Interventions, Comparators, and Outcomes}

We will not apply any restrictions regarding interventions, comparators, or outcomes.

\section{Types of Publications}

We will include observational and experimental studies, qualitative studies, and protocols that provide a working definition of pediatric CCI or prolonged PICU admission. Then, we will exclude literature reviews, unpublished literature, editorials, commentaries and opinion pieces, conference proceedings, abstracts, and books. Given the emerging nature and recognition of $\mathrm{CCI}$ in children, we will exclude records published before 1990. We will exclude studies that were not published in English or French.

\section{Search Strategy}

We developed a preliminary search strategy in two electronic databases (MEDLINE and CINAHL) and piloted this in consultation with a health research librarian (RC). We developed the final search strategy in MEDLINE, which was peer-reviewed by 2 additional health research librarians not involved in the study, and then translated it into the other databases, as appropriate (Textbox 1). We will search four databases that index citation titles or abstracts using English Medical Subject 
Headings terms and keywords from their dates of inception to March 2021: Ovid MEDLINE, Embase, CINAHL, and Web of to identify any studies that may have avoided the final database search.

Textbox 1. Search strategy (MEDLINE). adj: adjacent; epub: electronic publication; exp: explode; .mp: multi-purpose; PICU: pediatric intensive care unit.

Database

- Ovid MEDLINE epub ahead of print, in-process and other nonindexed citations, Ovid MEDLINE(R) daily and Ovid MEDLINE(R) 1946-present

\section{Search strategy}

- intensive care units/and (child* or pediatric or paediatric).mp. [mp=title, abstract, original title, name of substance word, subject heading word, floating sub-heading word, keyword heading word, organism supplementary concept word, protocol supplementary concept word, rare disease supplementary concept word, unique identifier, synonyms]

- Intensive care units, pediatric/

- PICU.mp.

- ((p?ediatric* or child or children*) adj3 (acute* or critical* or intens*)).mp.

- $\quad$ or $11-4$

- $\quad \exp$ Critical Care/

- Critical Illness/

- (critical* or intens*).mp. [mp=title, abstract, original title, name of substance word, subject heading word, floating sub-heading word, keyword heading word, organism supplementary concept word, protocol supplementary concept word, rare disease supplementary concept word, unique identifier, synonyms]

- $\quad$ or/6-8

- $\quad$ exp chronic disease/

- length of stay

I

- ((long or duration or length) adj3 (stay or hospitali*)).mp. [mp=title, abstract, original title, name of substance word, subject heading word, floating sub-heading word, keyword heading word, organism supplementary concept word, protocol supplementary concept word, rare disease supplementary concept word, unique identifier, synonyms]

- $\quad$ or/10-12

- 5 and 9 and 13

- ((chronic* or persist* or long term or longterm or long-stay or prolong* or protract* or extend* or extensive or lengthy or difficult*) adj5 (acute* or critical* or intens* or ill or illness* or sick or sickness* or care)).mp. [mp=title, abstract, original title, name of substance word, subject heading word, floating sub-heading word, keyword heading word, organism supplementary concept word, protocol supplementary concept word, rare disease supplementary concept word, unique identifier, synonyms]

- 5 and 15

- 14 or 16

- ((p? ediatric* or child or children*) adj5 (chronic* or persist* or long term or longterm or prolong* or protract* or extend* or extensive or lengthy or difficult* or ((long or duration) adj3 stay)) adj5 (acute* or critical* or intens* or ill or illness* or sick or sickness* or care)).mp.

- 17 or 18

\section{Study Selection}

\section{Search Strategy and Study Selection Criteria Piloting}

The team used an iterative approach to evaluate and refine the preliminary search strategy and study selection criteria. Using the results of the preliminary search strategy, 4 members of the core study team independently reviewed an initial set of 100 randomly selected citations using the initial study selection criteria. Each record was reviewed in triplicate. We screened the 100 citations in two steps (title and abstract, then full text), discussed discrepancies, and refined the eligibility criteria. The lead investigator (DZ) reviewed the reference lists of studies meeting all inclusion criteria, identified any relevant studies, and, together with the health sciences librarian, refined the search strategy if these relevant studies were missed by the database search. Following this initial round, we reevaluated the revised study selection criteria using a second set of 100 random citations assessed independently and in triplicate. The conflict rates were $45.5 \%$ (5/11 full texts) and 7.7\% (1/13 full texts) in full-text assessment during the two iterative piloting rounds. Following these two iterative piloting rounds, the team established a consensus on the study selection criteria. A total of 8 eligible studies were identified during the piloting. 


\section{Crowdsourcing}

Given the large number of citations identified in the final search strategy, we will use a hybrid approach comprising crowdsourcing and a machine learning (ML) algorithm to expedite the screening of records. The crowdsourcing methodology for systematic reviews has been previously validated $[19,20]$ and used in a variety of health research reviews to accelerate the citation screening and provide more timely research output, while still allowing for rigorous review conduct [21-23]. We will recruit a curated crowd of approximately 30 English- and French-speaking reviewers with content and methodological expertise from international PICU networks (eg, Canadian Critical Care Trials Group, Pediatric Acute Lung Injury, and Sepsis Investigators group), email, social media (using the hashtags \#PedsICU, \#PICSp, and \#CCI), and a dedicated study crowdsourcing event page on insightScope [24]. Authorship incentives will be offered to crowd reviewers who achieved specific screening milestones (ie, group authorship if $\geq 500$ abstracts and $\geq 50$ full texts screened, named authorship if $\geq 1000$ abstracts, $\geq 100$ full texts screened, and participated in data abstraction).

Before formal screening, prospective reviewers will be provided with a copy of the protocol and selection criteria. Prospective reviewers will first perform screening on a test set designed using the piloted study selection criteria [25]. The test set will contain 100 citations from the pilot phase with 10 eligible (true positive) citations. Prospective reviewers must achieve a sensitivity of $\geq 80 \%$ before they are given access to the full set of study records. Reviewers who do not achieve $\geq 80 \%$ sensitivity will be provided with additional training before being given access to the full set of study records.

We will use a dedicated channel on Slack (Slack Technologies), a cloud-based team communication platform, to streamline the study progress updates and reviewer communication [26,27].

\section{Algorithm}

ML algorithms are being increasingly used to assist in citation screening for systematic reviews, particularly in large reviews [28-31]. We will develop an ML algorithm to semiautomate citation screening for this scoping review at the title and abstract stage only, which is consistent with previously described approaches (Figure 1) [31]. The independent and duplicate screening of at least 4000 citations through to the full text by crowd members will constitute a training set that we will use to evaluate five ML algorithms (bag of words, term frequency-inverse document frequency, word to vector, document to vector, and fast text). These algorithms assess the citation title and abstract (where available) and rank each citation by relevance based on the text captured in the study selection criteria and project goal, with the highest ranking citations being retained based on a threshold set by the investigator (eg, a threshold of $70 \%$ would retain the $30 \%$ highest ranking citations). The titles and abstracts of citations from the four electronic databases were downloaded in English; therefore, no language adaptations were required to apply the ML algorithms to non-English-language studies.

Figure 1. Integration of crowdsourcing and machine learning in the scoping review.

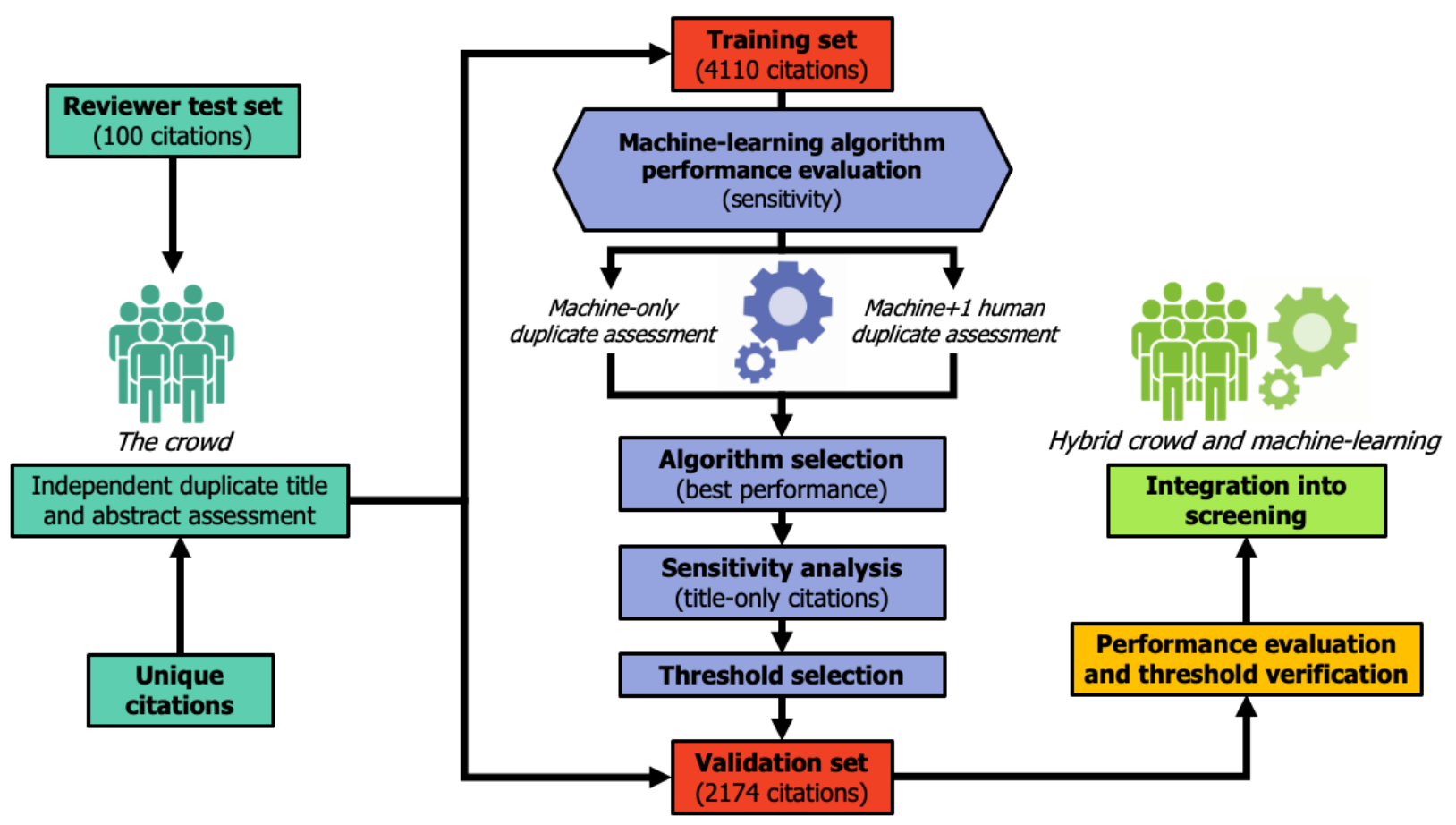

We will select the two highest performing algorithms from the training set and evaluate their sensitivity and specificity at a variety of thresholds, when used alone and in combination with a single human reviewer. We will also separately evaluate the performance of the two highest performing ML algorithms for citations without an abstract (ie, title only) to evaluate whether a unique threshold would be required. For both ML algorithms, we will determine the threshold at which the sensitivity is $>95 \%$ when used in combination with a single human reviewer. This 
approach is consistent with the individual sensitivity of expert reviewers, as described in previous studies [20,23,32,33].

Once developed, we will evaluate the performance of the two candidate ML algorithms on an additional validation set constituting at least 2000 citations screened independently in duplicate by crowd members. Our a priori methodology will be to proceed with the duplicate independent human assessment of citations above the selected threshold score, and machine plus one independent human assessment for citations below the threshold score. We will also plan to apply an additional lower threshold score if the sensitivity data for the candidate ML algorithms consistently exceed our sensitivity goal (ie, 95\%). This lower threshold will serve to exclude the most irrelevant citations through assessment by the ML algorithm alone.

\section{Integration of Hybrid Crowdsourcing and ML Algorithm Citation Screening}

The integration of crowdsourcing and ML algorithm methods into citation screening in this scoping review is outlined in Figure 1. We will download records from the electronic search into Endnote for duplicate removal and export the citation list for screening to insightScope [34], a platform for executing large reviews through crowdsourcing. We will upload citation abstracts and full-text articles with inclusion and exclusion criteria for insightScope. Screening will be performed in two steps (title and abstract, then full text) against the inclusion criteria by 2 independent reviewers. We will record reasons for the exclusion of citations excluded from full-text screening. As previously described, no language adaptations to the screening process for non-English studies will be required for the title and abstract stage, as citations retrieved from electronic databases are in English. However, full texts in French will be reviewed independently and in duplicate by French-speaking crowd reviewers. All screening conflicts (either between 2 humans or a machine and 1 human) will be resolved by third-party adjudication by the members of the core study team, as required.

\section{Data Charting}

We will perform data abstraction using the piloted electronic data abstraction forms created in insightScope. The data abstraction forms were created by one investigator (DZ) and piloted by the members of the core investigative team (JDM, $\mathrm{BR}, \mathrm{NP}, \mathrm{KO}$, and $\mathrm{KC}$ ) against a total of 8 eligible studies. We have described the data items in Textbox 2. Before formal data abstraction, we will provide all data abstractors with training (ie, a data abstraction manual and training video). Data will be abstracted by 2 independent reviewers from the crowd, both independently and in duplicate. We will abstract data from the full-text publication and any related publications, referenced published protocols, or supplementary materials. Where necessary, one reviewer will extract graphical data using SourceForge Plot Digitizer, which will be checked by the second reviewer for accuracy. Moreover, where necessary, data will be abstracted from publications in French by French-speaking crowd reviewers independently and in duplicate. The study lead (DZ) resolved conflicts in data abstraction, as required. In the event of missing or unclear data related to our outcomes of interest, we will make a maximum of three attempts to contact the study authors for clarification. 
Textbox 2. Data items.

\section{Study characteristics}

- Author name and contact information

- Title

- Country of origin

- Journal and year of publication

- $\quad$ Study design

- Clinical setting and type of pediatric intensive care unit (eg, medical-surgical, cardiac only, and neuro-pediatric intensive care unit)

- Participant inclusion and exclusion criteria

- Total patients included

- $\quad$ Study period (dates)

\section{Study population definition}

- Definition of pediatric chronic critical illness (eg, as defined by study or referenced from another publication)

- Definition of prolonged pediatric intensive care unit or long-stay admission (eg, duration, as defined by study or referenced from another publication)

- If and how the definition was developed or validated by the primary study

- Prevalence of study participants with chronic critical illness or prolonged pediatric intensive care unit admission, as applicable to the study

Study population demographics and characteristics

- $\quad$ Age and sex

- Reason for pediatric intensive care unit admission

- Source of pediatric intensive care unit admission (eg, emergency department, neonatal intensive care unit, floor or step-down unit)

- Functional status characteristics (using validated tools, as categorized by the article)

- Severity of illness characteristics (using validated tools, as categorized by the article)

- Comorbidity and medical complexity status, including if and how patient medical complexity and comorbidity was described in the study

- Prevalence and types of organ support technologies in study participants (eg, mechanical ventilation, feeding support, circulatory support [vasoactive drugs, extracorporeal membrane oxygenation, ventricular assist device], and extrarenal filtration)

- Types of study participants (eg, children with chronic critical illness or prolonged pediatric intensive care unit admission, families, siblings, and health care providers)

\section{Outcomes evaluated}

- Stated primary outcome, including how it was measured and result

- Patient outcomes, including mortality (pediatric intensive care unit, hospital, and overall), discharge disposition (eg, high-dependency unit, ward, rehabilitation facility, and home), and health-related quality of life

- Family and sibling outcomes (any, as categorized by the article)

- Health care provider outcomes (any, as categorized by the article)

- Health care system outcomes, including the length of stay (pediatric intensive care unit and hospital), pediatric intensive care unit bed-day use or consumption, pediatric intensive care unit readmission rate or occurrence, and pediatric intensive care unit cost analyses

\section{Results Synthesis}

We will report data related to study characteristics descriptively using counts with the percentages or measures of central tendency and variance (eg, means/medians with SDs/IQR), as appropriate. We will use tables to narratively summarize data related to study population definitions, including the prevalence of the population studied (if applicable) and contextual variables related to study type, setting, and evaluated patient population. We will describe the important elements of the methodology used to derive the case definition of CCI and prolonged PICU admission, including but not limited to the size of study, study design, setting(s), and if criteria for agreement or convergence established a priori. We will group included studies into one of the two definition domains based on their explicitly identified study population of interest (ie, CCI or prolonged PICU admission) and summarize data for each, separately. We plan to categorize patient- and family-based outcomes evaluated in the included studies according to the domains of the PICU Core Outcome Set [35] (ie, overall health, cognitive function, physical 
function, and emotional function), as applicable, to help formulate a priority agenda for future research.

Statistical analyses will be performed using SPSS Statistics, version 26 (IBM), as necessary. We will not perform any meta-analyses of epidemiological or outcome data collected from primary publications, in keeping with the descriptive nature of this scoping review. In keeping with a scoping review methodology, we will not complete the risk of bias assessment for included studies or undertake the certainty of evidence assessment for this scoping review [13,14]. However, the limitations of the nature and extent of populations and outcomes evaluated in current pediatric CCI research will be addressed in the Discussion section of the paper.

\section{Results}

Database search, citation screening, and the data abstraction phases of this scoping review started on March 3, 2021, and were completed on April 16, 2021. Data verification is ongoing, with data analysis as follows: the analysis of the review, with results, is anticipated to be completed by fall 2021 .

\section{Discussion}

\section{Crowdsourcing and ML Algorithm Methods}

A total of 32 crowdsourced reviewers completed the test set of 100 citations, achieving a mean sensitivity of 91.6\% (SD 0.09).
Two reviewers with exactly $70 \%$ sensitivity on the test set were provided additional training on the study protocol and study selection criteria before citation screening. Of these, 28 reviewers, with a test set sensitivity of $92.1 \%$ (SD 0.09), participated in the citation screening. Reviewers originated from 11 countries and 5 continents.

As a prerequisite to incorporate an ML algorithm into citation screening, we determined the optimal algorithm and sensitivity threshold for operationalization. The sensitivities of the five evaluated ML algorithms when used alone or in combination with a single human reviewer to assess citations from the training set are presented in Figures 2 and 3, respectively. The 4110-citation training set included 28 citations meeting the inclusion criteria following an assessment by 2 reviewers after full-text review (ie, true positives). The two highest performing ML algorithms were bag of words and term frequency-inverse document frequency, demonstrating $93 \%$ and $100 \%$ sensitivity, respectively, at a threshold of $80 \%$ when citation assessments were performed by the ML algorithm alone. The sensitivities for both these ML algorithms were $100 \%$ at a threshold of $80 \%$ when citation assessments were performed by the ML algorithm in combination with a single human reviewer.

Figure 2. Machine learning algorithm training set performance (machine-only citation assessment). The bag-of-words and term frequency-inverse document frequency demonstrate the highest sensitivities up to a threshold of $80 \%$.

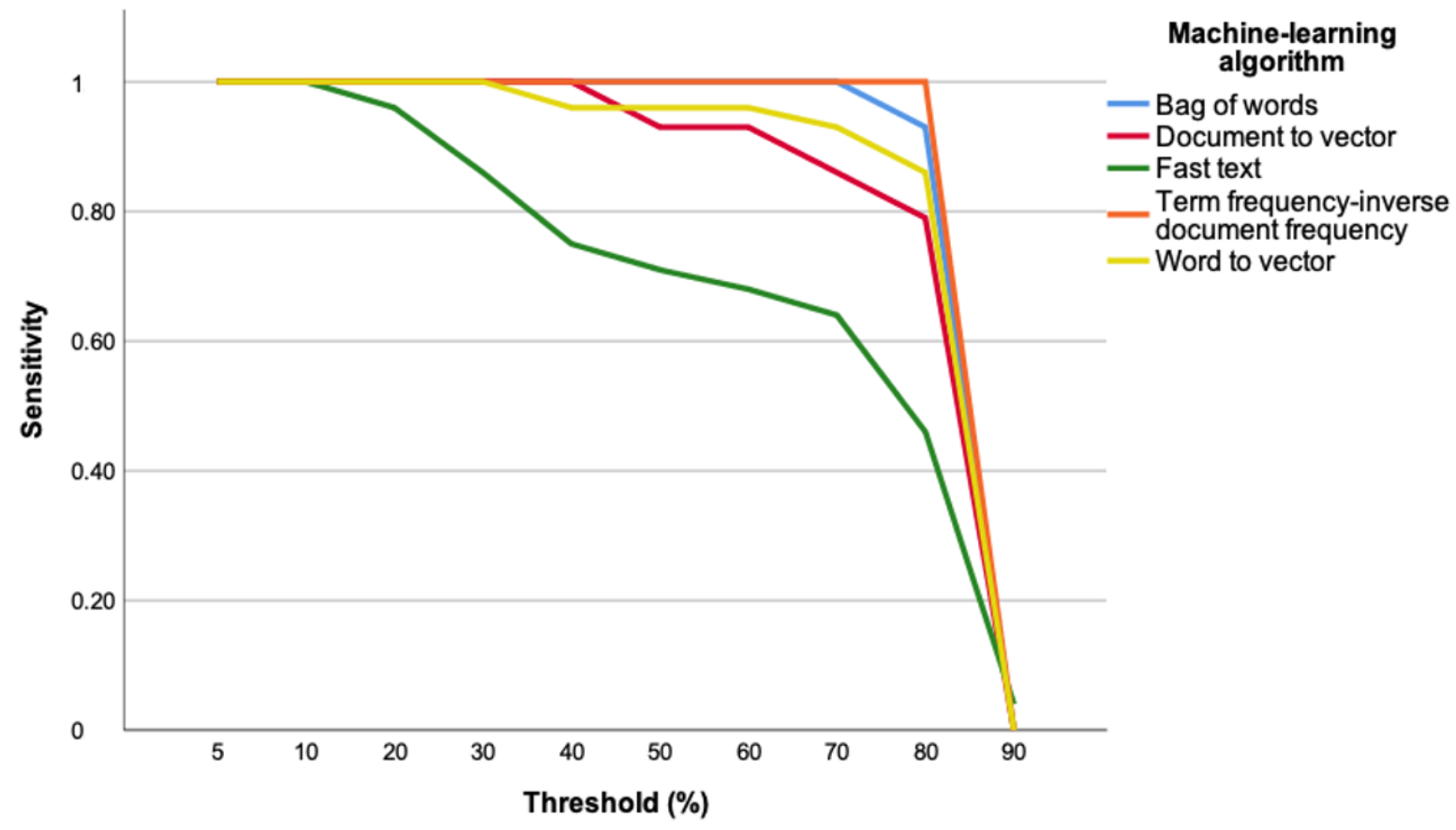


Figure 3. Machine learning algorithm training set performance (machine+one human reviewer citation assessment). The document to vector line overlaps with term frequency-inverse document frequency. The bag-of-words line overlaps with term frequency-inverse document frequency, demonstrating a sensitivity of $100 \%$ at a threshold of $80 \%$.

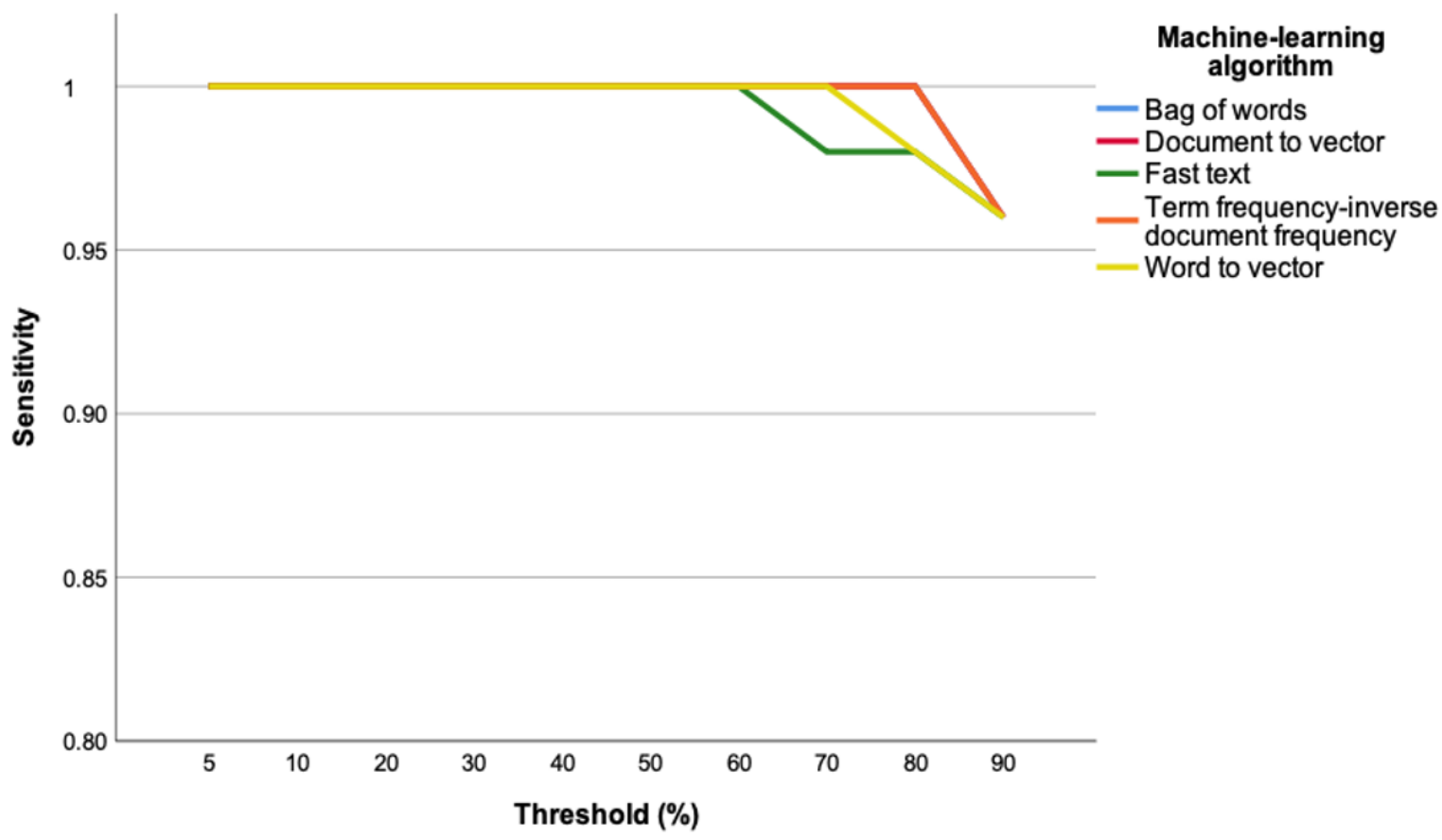

Additional sensitivity analyses were performed using the bag of words and term frequency-inverse document frequency algorithms using a separate threshold for citations without an abstract (ie, title only) to evaluate whether these citations perform differently. For this analysis, the threshold for citations with an abstract was fixed at $70 \%$, and the threshold for citations without an abstract varied among 30\%, 50\%, and 70\%. The bag of words and term frequency-inverse document frequency algorithms demonstrated sensitivities of $100 \%$ for all dual threshold combinations (ie, 70/30, 70/50, and 70/70), both when citations were assessed by the ML algorithm alone or in combination with a single human reviewer.

We subsequently evaluated the bag of words and term frequency-inverse document frequency ML algorithms on a validation set of 2174 additional citations. Again, these citations were screened independently and in duplicate by crowd reviewers. The validation set included nine unique citations that met the inclusion criteria. On the basis of the sensitivity results from the training set, we chose to apply the following conservative thresholds to evaluate performance on the validation set: $70 \%$ for citations with an abstract and $50 \%$ for citations with title only. Both the bag of words and term frequency-inverse document frequency algorithms demonstrated a sensitivity of $92 \%$ when citations were assessed using the ML algorithm alone, and a sensitivity of $100 \%$ when used in combination with a single human reviewer.

In addition to sensitivity, we evaluated the specificity of the ML algorithm. Both the term frequency-inverse document frequency and bag of words algorithms demonstrated a similar specificity at $70 \%$ threshold (ie, 0.68), but the term frequency-inverse document frequency algorithm retained three fewer false positive citations. Given this marginally better performance, term frequency-inverse document frequency was selected as the final ML algorithm. Considering that ML algorithms are relatively novel in the conduct of large scoping reviews, we adopted a conservative approach to integrating the algorithm into citation screening for the remaining citations in the review. For citations with an abstract, the following three thresholds were selected:

1. Citations with a score $\geq 70 \%$ threshold were assessed by duplicate independent human assessment.

2. Citations with a score between $30 \%$ and $70 \%$ threshold were assessed by machine plus one independent human assessment.

3. Citations with a score $\leq 30 \%$ threshold were assessed by machine-only assessment.

For citations without an abstract (ie, title only), we adopted a conservative approach by selecting a $50 \%$ threshold and no option for machine-only citation assessment. Therefore, citations with a score $\geq 50 \%$ threshold were assessed by duplicate independent human assessment, and citations with a score $<50 \%$ threshold were assessed by machine plus one independent human assessment.

\section{Strengths and Limitations}

This scoping review is the first phase of a larger research program to systematically evaluate children with CCI. To our knowledge, this scoping review is the first evidence synthesis to provide a systematic overview of the definitions used in the literature for identifying children with CCI and prolonged PICU admission. As such, the results of this review will be used to inform the development of a consensus case definition for pediatric CCI and set a priority agenda for future research. Defining pediatric CCI is an essential first step in understanding 
the epidemiology of this high-risk PICU population, and a prerequisite for conducting future interventional and outcomes research. As the aims of this scoping review are descriptive and exploratory in nature, this preliminary study will identify the potential need to conduct a systematic review to address targeted and explanatory epidemiologic questions. This scoping review will also demonstrate the feasibility and validity of two innovative evidence synthesis methods, crowdsourcing and an ML algorithm, to execute a large scoping review.

This review has several important limitations. As the goal of this scoping review was to describe the definitions of pediatric CCI and prolonged PICU admission, it is limited to studies that explicitly identified and defined these concepts. This review will potentially miss records that did not use this specific language to define their population, and excluded studies that did not provide or reference a definition of pediatric CCI or prolonged PICU admission. Similarly, the study selection criteria in this review will exclude studies that focused only on the concept of prolonged technology use (eg, prolonged mechanical ventilation, prolonged extracorporeal membrane oxygenation).
We seek to broadly understand pediatric CCI, and as a part of this objective, we will describe how the concept of organ support technology is applied in the published definitions of pediatric CCI.

\section{Conclusions}

This scoping review is the first, to the best of our knowledge, to (1) provide a systematic overview of the definitions used in the literature for identifying children with $\mathrm{CCI}$ and prolonged PICU admission and (2) describe the demographic and clinical characteristics of the populations historically defined in the pediatric CCI literature. This comprehensive literature review will evaluate existing or suggested definitions of pediatric CCI. In the absence of definitions, the review results will be used in future research to identify the key terms and constructs to inform the development of a working definition of pediatric CCI. Defining pediatric CCI is an essential first step in understanding the epidemiology of this high-risk PICU population and a prerequisite for conducting future interventional and outcomes research.

\section{Acknowledgments}

The authors would like to express their appreciation to Christine Neilson, Master of Library and Information Science (University of Manitoba), and Dr Margaret Sampson, Master of Library and Information Science (Children's Hospital of Eastern Ontario) for providing peer review of our MEDLINE search strategy. The authors also express their gratitude to Sanket Patel (Team Lead, insightScope) and Ravichandra Rachamalla (Machine Learning Development, insightScope) for their work related to the development, validation, and integration of machine learning algorithms. This scoping review did not receive any specific funding. DJZ is supported by the Canadian Institutes of Health Research Canada Graduate Scholarship_-Master's Award (award number $\mathrm{n} / \mathrm{a}$ ). The funders had no role in the study design, data collection and analysis, decision to publish, or preparation of the protocol.

\section{Authors' Contributions}

DJZ and KC conceived the idea for the scoping review. All authors contributed to the design of this review protocol. All authors participated in piloting and refining the search strategy, selection criteria, and data abstraction forms. DJZ drafted the paper. All authors read the paper, provided feedback, and approved the paper for submission. KC is the guarantor for this review.

\section{Conflicts of Interest}

None declared.

\section{References}

1. Namachivayam P, Shann F, Shekerdemian L, Taylor A, van Sloten I, Delzoppo C, et al. Three decades of pediatric intensive care: who was admitted, what happened in intensive care, and what happened afterward. Pediatr Crit Care Med 2010 Sep;11(5):549-555. [doi: 10.1097/PCC.0b013e3181ce7427] [Medline: 20124947]

2. Chan T, Rodean J, Richardson T, Farris RW, Bratton SL, Di Gennaro JL, et al. Pediatric critical care resource use by children with medical complexity. J Pediatr 2016 Oct;177:197-203. [doi: 10.1016/j.jpeds.2016.06.035] [Medline: 27453367]

3. Pinto NP, Rhinesmith EW, Kim TY, Ladner PH, Pollack MM. Long-term function after pediatric critical illness: results from the survivor outcomes study. Pediatr Crit Care Med 2017 Mar;18(3):122-130. [doi: 10.1097/PCC.0000000000001070] [Medline: 28107265]

4. Shapiro MC, Henderson CM, Hutton N, Boss RD. Defining pediatric chronic critical illness for clinical care, research, and policy. Hosp Pediatr 2017 Apr;7(4):236-244. [doi: 10.1542/hpeds.2016-0107] [Medline: 28351944]

5. Edwards JD, Houtrow AJ, Vasilevskis EE, Rehm RS, Markovitz BP, Graham RJ, et al. Chronic conditions among children admitted to U.S. pediatric intensive care units: their prevalence and impact on risk for mortality and prolonged length of stay*. Crit Care Med 2012 Jul;40(7):2196-2203 [FREE Full text] [doi: 10.1097/CCM.0b013e31824e68cf] [Medline: 22564961]

6. Namachivayam SP, Alexander J, Slater A, Millar J, Erickson S, Tibballs J, Paediatric Study Group and Australian and New Zealand Intensive Care Society. Five-year survival of children with chronic critical illness in Australia and New Zealand. Crit Care Med 2015 Sep;43(9):1978-1985. [doi: 10.1097/CCM.0000000000001076] [Medline: 25962079] 
7. Naghib S, van der Starre C, Gischler SJ, Joosten KF, Tibboel D. Mortality in very long-stay pediatric intensive care unit patients and incidence of withdrawal of treatment. Intensive Care Med 2010 Jan;36(1):131-136. [doi: 10.1007/s00134-009-1693-z] [Medline: 19855954]

8. Choong K, Fraser D, Al-Harbi S, Borham A, Cameron J, Cameron S, et al. Functional recovery in critically ill children, the "WeeCover" multicenter study. Pediatr Crit Care Med 2018 Feb;19(2):145-154. [doi: 10.1097/PCC.0000000000001421] [Medline: 29394221]

9. Kalzén H, Larsson B, Eksborg S, Lindberg L, Edberg KE, Frostell C. Survival after PICU admission: the impact of multiple admissions and complex chronic conditions. PLoS One 2018 Apr 5;13(4):e0193294 [FREE Full text] [doi: 10.1371/journal.pone.0193294] [Medline: 29621235]

10. Henderson CM, Williams EP, Shapiro MC, Hahn E, Wright-Sexton L, Hutton N, et al. "Stuck in the ICU": caring for children with chronic critical illness. Pediatr Crit Care Med 2017 Nov;18(11):561-568. [doi:

10.1097/PCC.0000000000001332] [Medline: 28922265]

11. Donohue PK, Williams EP, Wright-Sexton L, Boss RD. "It's Relentless": providers' experience of pediatric chronic critical illness. J Palliat Med 2018 Jul;21(7):940-946. [doi: 10.1089/jpm.2017.0397] [Medline: 29570427]

12. Rose L, Istanboulian L, Allum L, Burry L, Dale C, Hart N, et al. Patient and family centered actionable processes of care and performance measures for persistent and chronic critical illness: a systematic review. Crit Care Explor 2019 Apr 17;1(4):e0005 [FREE Full text] [doi: 10.1097/CCE.0000000000000005] [Medline: 32166252]

13. Peters MD, Marnie C, Tricco AC, Pollock D, Munn Z, Alexander L, et al. Updated methodological guidance for the conduct of scoping reviews. JBI Evid Synth 2020 Oct;18(10):2119-2126. [doi: 10.11124/JBIES-20-00167] [Medline: 33038124]

14. Munn Z, Peters MD, Stern C, Tufanaru C, McArthur A, Aromataris E. Systematic review or scoping review? Guidance for authors when choosing between a systematic or scoping review approach. BMC Med Res Methodol 2018 Nov 19;18(1):143 [FREE Full text] [doi: 10.1186/s12874-018-0611-x] [Medline: 30453902]

15. Arksey H, O'Malley L. Scoping studies: towards a methodological framework. Int J Soc Res Methodol 2005 Feb;8(1):19-32. [doi: 10.1080/1364557032000119616]

16. Levac D, Colquhoun H, O'Brien KK. Scoping studies: advancing the methodology. Implement Sci 2010 Sep 20;5:69. [doi: 10.1186/1748-5908-5-69] [Medline: 20854677]

17. Tricco AC, Lillie E, Zarin W, O'Brien KK, Colquhoun H, Levac D, et al. PRISMA extension for Scoping Reviews (PRISMA-ScR): checklist and explanation. Ann Intern Med 2018 Oct 02;169(7):467-473 [FREE Full text] [doi: 10.7326/M18-0850] [Medline: 30178033]

18. Zorko D, McNally J, Rochwerg B, Pinto N, Couban R, O'Hearn K. Pediatric chronic critical illness: a protocol for a scoping review. OSF Preprints 2021 (forthcoming). [doi: 10.31219/osf.io/umr8x]

19. Nama N, Sampson M, Barrowman N, Sandarage R, Menon K, Macartney G, et al. Crowdsourcing the citation screening process for systematic reviews: validation study. J Med Internet Res 2019 Apr 29;21(4):e12953 [FREE Full text] [doi: 10.2196/12953] [Medline: 31033444]

20. Nama N, Iliriani K, Xia MY, Chen BP, Zhou LL, Pojsupap S, et al. A pilot validation study of crowdsourcing systematic reviews: update of a searchable database of pediatric clinical trials of high-dose vitamin D. Transl Pediatr 2017 Jan;6(1):18-26 [FREE Full text] [doi: 10.21037/tp.2016.12.01] [Medline: 28164026]

21. Tricco AC, Garritty CM, Boulos L, Lockwood C, Wilson M, McGowan J, et al. Rapid review methods more challenging during COVID-19: commentary with a focus on 8 knowledge synthesis steps. J Clin Epidemiol 2020 Oct;126:177-183 [FREE Full text] [doi: 10.1016/j.jclinepi.2020.06.029] [Medline: 32615209]

22. Ranard BL, Ha YP, Meisel ZF, Asch DA, Hill SS, Becker LB, et al. Crowdsourcing--harnessing the masses to advance health and medicine, a systematic review. J Gen Intern Med 2014 Jan;29(1):187-203 [FREE Full text] [doi: 10.1007/s11606-013-2536-8] [Medline: 23843021]

23. Ashkanase J, Nama N, Sandarage RV, Penslar J, Gupta R, Ly S, et al. Identification and evaluation of controlled trials in pediatric cardiology: crowdsourced scoping review and creation of accessible searchable database. Can J Cardiol 2020 Nov;36(11):1795-1804. [doi: 10.1016/j.cjca.2020.01.028] [Medline: 32330435]

24. Zorko D. Chronic critical illness in children: A scoping review. insightScope. URL: https://insightscope.tech/preview project/ $\underline{217}$ [accessed 2021-05-17]

25. Nama N, Barrowman N, O'Hearn K, Sampson M, Zemek R, McNally JD. Quality control for crowdsourcing citation screening: the importance of assessment number and qualification set size. J Clin Epidemiol 2020 Jun;122:160-162. [doi: 10.1016/i.jclinepi.2020.02.009] [Medline: 32142884]

26. Gofine M, Clark S. Integration of Slack, a cloud-based team collaboration application, into research coordination. J Innov Health Inform 2017 Jun 30;24(2):936 [FREE Full text] [doi: 10.14236/jhi.v24i2.936] [Medline: 28749322]

27. Johnson HA. Slack. J Med Libr Assoc 2018 Jan 12;106(1):148-151. [doi: 10.5195/jmla.2018.315]

28. Marshall IJ, Wallace BC. Toward systematic review automation: a practical guide to using machine learning tools in research synthesis. Syst Rev 2019 Jul 11;8(1):163 [FREE Full text] [doi: 10.1186/s13643-019-1074-9] [Medline: 31296265]

29. Tsou AY, Treadwell JR, Erinoff E, Schoelles K. Machine learning for screening prioritization in systematic reviews: comparative performance of Abstrackr and EPPI-Reviewer. Syst Rev 2020 Apr 02;9(1):73 [FREE Full text] [doi: 10.1186/s13643-020-01324-7] [Medline: $\underline{\text { 32241297] }}$ 
30. Gates A, Gates M, Sebastianski M, Guitard S, Elliott SA, Hartling L. The semi-automation of title and abstract screening: a retrospective exploration of ways to leverage Abstrackr's relevance predictions in systematic and rapid reviews. BMC Med Res Methodol 2020 Jun 03;20(1):139 [FREE Full text] [doi: 10.1186/s12874-020-01031-w] [Medline: 32493228 ]

31. Bannach-Brown A, Przybyła P, Thomas J, Rice AS, Ananiadou S, Liao J, et al. Machine learning algorithms for systematic review: reducing workload in a preclinical review of animal studies and reducing human screening error. Syst Rev 2019 Jan 15;8(1):23 [FREE Full text] [doi: 10.1186/s13643-019-0942-7] [Medline: 30646959]

32. Nama N, Hennawy M, Barrowman N, O'Hearn K, Sampson M, McNally JD. Successful incorporation of single reviewer assessments during systematic review screening: development and validation of sensitivity and work-saved of an algorithm that considers exclusion criteria and count. Syst Rev 2021 Apr 05;10(1):98 [FREE Full text] [doi: 10.1186/s13643-021-01632-6] [Medline: 33820560]

33. Noel-Storr AH, Redmond P, Lamé G, Liberati E, Kelly S, Miller L, et al. Crowdsourcing citation-screening in a mixed-studies systematic review: a feasibility study. BMC Med Res Methodol 2021 Apr 26;21(1):88 [FREE Full text] [doi: 10.1186/s12874-021-01271-4] [Medline: 33906604]

34. The future of systematic reviews. insightScope. URL: https://insightscope.ca [accessed 2021-05-17]

35. Fink E, Maddux A, Pinto N, Sorenson S, Notterman D, Dean J, Pediatric Outcomes STudies after PICU (POST-PICU) Investigators of the Pediatric Acute Lung Injury and Sepsis Investigators (PALISI) Network and the Eunice Kennedy Shriver National Institute of Child Health and Human Development Collaborative Pediatric Critical Care Research Network (CPCCRN). A core outcome set for pediatric critical care. Crit Care Med 2020 Dec;48(12):1819-1828. [doi: 10.1097/CCM.0000000000004660] [Medline: 33048905]
Abbreviations
CCI: chronic critical illness
ML: machine learning
PICU: pediatric intensive care unit

Edited by G Eysenbach; submitted 27.05.21; peer-reviewed by A Woodruff, E Mohammadi; comments to author 10.07.21; revised
version received 30.07.21; accepted 30.07.21; published 01.10.21
Please cite as:
Zorko D, McNally JD, Rochwerg B, Pinto N, Couban R, O'Hearn K, Choong K
Pediatric Chronic Critical Illness: Protocol for a Scoping Review
JMIR Res Protoc 2021;10(10):e30582
URL: $\underline{\text { https://www.researchprotocols.org/2021/10/e30582 }}$
doi: $10.2196 / 30582$
PMID:

CDavid Zorko, James Dayre McNally, Bram Rochwerg, Neethi Pinto, Rachel Couban, Katie O’Hearn, Karen Choong. Originally published in JMIR Research Protocols (https://www.researchprotocols.org), 01.10.2021. This is an open-access article distributed under the terms of the Creative Commons Attribution License (https://creativecommons.org/licenses/by/4.0/), which permits unrestricted use, distribution, and reproduction in any medium, provided the original work, first published in JMIR Research Protocols, is properly cited. The complete bibliographic information, a link to the original publication on https://www.researchprotocols.org, as well as this copyright and license information must be included. 